\title{
Qualities, Relations, and Property Exemplification
}

\author{
Dale Jacquette
}

Received: 7 February 2011/Accepted: 24 May 2011/Published online: 10 June 2011

(C) Springer Science+Business Media B.V. 2011

\begin{abstract}
The question whether qualities are metaphysically more fundamental than or mere limiting cases of relations can be addressed in an applied symbolic logic. There exists a logical equivalence between qualitative and relational predications, in which qualities are represented as one-argument-place property predicates, and relations as more-than-one-argument-place predicates. An interpretation is first considered, according to which the logical equivalence of qualitative and relational predications logically permits us ontically to eliminate qualities in favor of relations, or relations in favor of qualities. If metaphysics is understood at least in part as an exercise in ontic economy, then we may be encouraged to adopt a property ontology of qualities without quality-irreducible relations, or relations without relation-irreducible qualities. If either strategy is followed, the choice of reducing qualities to relations or relations to qualities will need to be justified on extra-logical grounds. These might include a perceived greater intuitiveness, explanatory fecundity, compatibility with cognitive ontogeny or developmental psychology, expressive or explanatory elegance or cumbersomeness, and an openended list of philosophical motivations that could reasonably favor the ontic prioritization of qualities over relations or relations over qualities. Despite its intuitive appeal, the thesis that logical equivalence together with extra-logical preferences justifies unidirectional ontic reduction of relations to qualities or qualities to relations is rejected in light of the more defensible proposition that the logical equivalence of qualitative and relational predications actually supports the opposite conclusion, that both qualities and relations are logically indispensable to a complete ontology of properties. The logical equivalence of qualitative and relational predications, insofar as we continue to observe the distinction, makes it logically necessary ontically for both qualities and relations to exist whenever either one
\end{abstract}

\section{Jacquette $(\bowtie)$}

Institut für Philosophie, Lehrstuhl für theoretische Philosophie, Unitobler, University of Bern, Länggassstrasse 49a, 3000 Bern, Switzerland

e-mail: dale.jacquette@philo.unibe.ch 
exists. That logically equivalent qualitative and relational predications have as their truth-makers the exemplification by objects of both qualities and relations as equifoundational properties further suggests that there is no deeper logical distinction between qualities and relations, but only two convenient lexical-grammatical designations for property predications involving one- versus more-than-one-argumentplace.

Keywords Abstraction - Exemplification - Identity · Logic, logical equivalence · Metaphysics · Ontic reduction · Ontology · Property · Quality · Relation ·

Semantics

\section{Ontic Reductions}

We begin with a slate of questions. Is it possible, and what would it mean, to reduce qualities to relations and-or relations to qualities? What is the methodology by which an ontic reduction of qualities to relations (qualities as limiting case relations) or relations to qualities (relations as internally predicationally complex qualities) is supposed to be effected? What is the role of logical equivalence in supporting reductions of qualitative to relational or relational to qualitative predications? How does predicate syntactical economy generally relate to ontic economy? What should the logical equivalence of qualitative and relational predications be taken to imply concerning the ontology and exemplification of qualities and relations? Is the relation one-one, one-many, many-one, many-many? Are there distinct logical or other philosophical advantages to be sought in an ontic reduction that eliminates either the category of qualities or the category of relations from the metaphysics of properties and the semantics of property predications? What is to be gained, and what sacrifices might be expected, under the most severe ontic austerity measures? Given the choice, can we formulate a better properties ontology with only qualities to the exclusion of quality-irreducible relations, or with only relations to the exclusion of relation-irreducible qualities? How much can logic contribute to understanding and managing our metaphysical choices, and about which ontic matters is logic silent?

We consider an interesting popular interpretation of the logical equivalence of qualitative and relational predications. The logical equivalence exists, and the equivalent propositions satisfy the argument-place-number criterion for distinguishing qualitative and relational predications. Although, to be candid, some parts are only trivially or vacuously qualitative or relational in predicational form. The interpretation implies that logic offers metaphysics the option of accepting a more austere ontology of properties consisting only of qualities, excluding relations as a special condition of objects, or exclusively of relations without qualities, except as limiting cases of relations. Metaphysics goes beyond logic, but with logic's blessing, then, by advancing extra-logical reasons for preferring qualities over relations or relations over qualities.

We track this apparently reasonable assumption to see where it leads to suppose that the logical equivalence of qualitative and relational predications presents 
metaphysics with the option of reducing qualities exclusively to relations or relations exclusively to qualities. The logical equivalence of qualitative and corresponding relational predication transforms itself has the logical form of a biconditional, naturally consisting of two conditionals. One conditional, pointing in one direction, invites the syntactical reduction of relational to qualitative predications, and the other of qualitative to relational predications. Thus, we can say everything we need to say about relations in purely qualitative terms, and similarly for relational variants of anything we may want to say about qualities. If, given the logical equivalence of qualitative and relational predications, we need only find respectable extra-logical grounds for preferring qualities over relations or relations over qualities, then we must look to the question of what sort of extralogical arguments might be found or fashioned for reducing relations to qualities or qualities to relations in a more economical preferred property ontology.

We conclude in the end, despite the intuitive appeal of authorizing ontic reductions on the strength of logical equivalences, that there are even better reasons for rejecting the syntactical-to-ontic reduction principle, at least where the ontology of qualities and relations and the logic and semantics of qualitative and relational predications are concerned. The syntactical-to-ontic reduction principle is nevertheless sufficiently interesting to warrant careful critical consideration independently of the metaphysics of properties. The common 'Quinean' strategy of many logically trained analytic metaphysicians is to identify logical equivalences among expressions referring to propositions and properties, sets and properties, sets and propositions, mental and physical states, or the like, and then to interpret the equivalences as implying that theoretically we need only one of the logically equivalent propositions and its ontic truth-makers exclusively, without and rather than or in preference to another ontically redundant terminology. ${ }^{1}$

(I:1) SYNTACTICAL-TO-ONTIC REDUCTIONISM

$Q \leftrightarrow R$ implies that $R$-entities are ontically dispensable in deference to $Q$-entities, and conversely

An ontology of properties can logically be reduced from an ontic domain containing both $Q$ - and $R$ entities, to a domain containing only $Q$ - and no $R$-entities, or, alternatively, to a ontic domain containing only $R$ - and no $Q$-entities

The model is seen in, if not self-consciously borrowed from, classical logic, where it is similarly observed that we can eliminate either $\rightarrow$ in favor of $\vee$ and $\neg$, or $\vee$ in favor of $\rightarrow$ and $\neg$, on the strength of the logical equivalence, $[p \rightarrow q] \leftrightarrow$ $[\neg p \vee q]$. We thereby simplify a specific more minimal syntax than the usual generous supply of distinct albeit interdefineable truth functions. The important question is whether in this case we also thereby effect an ontic reduction in the number of truth functions. The philosophical problem is how properly to interpret

\footnotetext{
1 The interpretation of logical equivalence between qualitative and relational predication follows general Quinean paraphrastic protocols. See Quine (1960, esp. pp. 161, 180-188, 210, 221, 227-228, 250, 258-259). A paradigmatic example of Quine's method is found in Quine (1964, pp. 209-216), Lambert (1987).
} 
the full ontic implications entailed or approved by specific 'reductive' applications of the logical equivalence relation, $\leftrightarrow$. Are we right to think, if such a logical equivalence holds, then, since in that case we can always say whatever we need or want to say about one part of the logical equivalence exclusively in terms of the other logically equivalent part, that therefore we do not need both equivalencerelated concepts in theory or language? Are we otherwise then simply multiplying names for identical things beyond expressive or explanatory necessity? Or are we logically stuck, does logic thereafter demand that ontically we must always have both concepts represented by the two logically equivalent predications whenever we have either one or the other?

The essential connection between syntactical reform and corresponding ontic reduction is supposed to be that ontic commitments are made by thinkers when they accept the truth of theories that are in turn composed of propositions, whose truthmakers finally presuppose the existence of this or that object exemplifying this or that property. If we do not need to refer to a certain category of objects or properties in the true propositions constituting a theory, but can logically equivalently express whatever relevant truths apply by referring only to the properties of another category of objects in propositions involving a distinct vocabulary, then it is reasonable to conclude that we can reduce our ontic commitments to the category of objects referred to in whatever true propositions minimally logically imply both sets of propositions. If we can reduce our overall ontic commitment by accepting as true only those propositions that make reference to qualities exclusively rather than relations, or to relations rather than qualities, then perhaps we should try to do so. Not only are we obligated to work toward ontic reductions because it is a responsibility of metaphysics to keep philosophy's ontic house in good order, but, more importantly, as we proceed, for the sake of sharpening our sense of what may be truly fundamental and essential in analytic metaphysics.

The reliance on logical equivalence as a justification for ontic reduction is disputable. Reasoning from the reduction of a redundantly opulent to a more austere logical syntax toward a corresponding ontic reduction resulting in a comparatively more austere ontology is frequently taken for granted as a philosophical privilege to be exercised with discretion but at our convenience. We criticize this libertine expectation, and cast doubt on the prospects of achieving a warranted ontic reduction from any true logical equivalence. We conclude that the logical equivalence of qualitative and relational predications in particular does not logically imply the ontic reduction or collapsing of qualities and relations into a single category. We maintain that the logical equivalence of qualitative and relational predications implies instead that both qualities and relations must exist as two ontically distinct but logically equivalently expressible property subspecies. If this is the correct interpretation of the ontic implications of the logical equivalence of qualitative and relational predication syntactical transforms, then, merely on the strength of the logical equivalence of qualitative and relational predications, together with any supplementary extra-logical rationale, the foundations of metaphysics logically cannot be reduced to qualities without relations, or to relations without qualities, but qualities and relations logically are equally fundamental. 
Thus, we shall finally argue in support of a contrary interpretation of the logical equivalence of qualitative and relational predications:

(I:2) SYNTACTICAL-TO-ONTIC ANTI-REDUCTIONISM

$Q \leftrightarrow R$ implies that $R$-entities exist iff $R$-entities exist, and hence that neither is ontically dispensable in deference to the other

An ontology of properties logically cannot be reduced from an ontic domain containing both $Q$ - and $R$-entities, to a domain containing only $Q$ - and no $R$-entities, or, alternatively, to a ontic domain containing only $R$ - and no $Q$-entities

\section{Abstraction Equivalences for Qualitative and Relational Predications}

To provide an ontic reduction of qualities to relations, we begin with a stereotypical quality expression of the simplest predicational logical form, $F a$. Then we show how to reduce it by the method of $\lambda$-abstraction to produce a (vacuously) relational expression in logically equivalent form. ${ }^{2}$

We assume the following sense of syntactical to ontic reduction:

ONTIC REDUCTION $(O R)$

$\forall x, y\left[\operatorname{OR}(x)=y \leftrightarrow\left[x \neq y \wedge \forall F_{1} \exists F_{2}\left[F_{1} \neq F_{2} \wedge\left[F_{1}(x) \rightarrow F_{2}(y)\right]\right]\right]\right]$

The idea is that $x$ is ontically reducible to distinct $y$ iff any truth we want to express about $x$ logically implies in different terms a corresponding truth about $y$. The relevant application for the reduction of properties exclusively to qualities or relations is that in which $x$ is a quality (relation) and $y$ is a relation (quality), $F_{1}(x)$ is a qualitative (relational) predication, and $F_{2}(y)$ is a distinct relational (qualitative) predication. We show below that a similarly generalized ontic reductive transformation of qualitative to relational predications is also attainable. The same principle of ontic reduction, in two applications covering both reduction directions, implies not only that relations are ontically reducible to qualities, but that qualities are ontically reducible to relations.

We illustrate the use of $\lambda$-abstraction in conjunction with the principle of ontic reduction for the case in which proposition $p=$ Object a has quality $F$ and object $a$ has quality $G$; in elementary predicate logic, $p=F a \wedge G a$. Where $p$ is an open sentence with every object variable $x$ unbound, the $\lambda$-abstract of $p$, $\lambda x[p]=\lambda x[F x \wedge G x] a$. Now instead of predicating property $F$ and property $G$ of object $a$, in a truth functional conjunction of separate predications, we can attach in

\footnotetext{
${ }^{2}$ See note 5 below. Standard solutions are to restrict iterative syntax constructions by type theory orderings, to wffs already derived within a proof structure from logical theorems, or by making $\lambda$-abstraction conditional on the non-implication of syntactical inconsistency.
} 
one single predication the $\lambda$-abstracted property of being $F$-and- $G$ of object $a$. Predicate abstraction now permits logical equivalences to be expressed between relations and qualities. Using $\lambda$-abstraction, relation terms can be logically equivalently expressed exclusively by means of quality terms, and conversely. ${ }^{3}$

Qualities are properties expressed as one-argument-place predicates, as when we write $F a$, saying that object $a$ has quality $F$. Relations are properties expressed by more-than-one-argument-place predicates, as when we write, Rab or $a R b$, meaning that object $a$ stands in relation $R$ to object $b$. Nor is there any reason to suppose that two-place relations exhaust the field. Between-ness requires three terms, as do many other relations, and indefinitely more argument place relational terms might be needed, even for the logic of everyday relational concepts, such as being the cousin of the nephew of an uncle of the sister of an aunt. As Wittgenstein rightly remarks, asking rhetorically in Tractatus Logico-Philosophicus 5.5541: 'How could we decide a priori whether, for example, I can get into a situation in which I need to symbolize with a sign of a 27 -termed relation? ${ }^{4}$

A naïve formulation for $\lambda$-abstraction equivalence in the predicationally most basic case states, schematically: $\forall x[[\ldots x \ldots] \leftrightarrow \lambda y[\ldots y \ldots] x]$. As a topic for another occasion, abstraction is so powerful that restrictions must be placed on its naïve formulation to avoid the abstraction of logically self-contradictory predicates. ${ }^{5}$ Type-related syntax restrictions that forbid abstraction from such paradoxical symbol combinations as $x x$ or $F F$ (since object $x$ cannot fail to be a property $F$ ), are already in force in classical predicate-quantificational logic, and in any case they do not apply to the elementary first-order logic to which we confine immediate attention. Equally, we can restrict $\lambda$-abstraction to formulas that have appeared within the course of a derivation, and are not merely chosen at random as merely constructible wffs (well-formed formulas) or permissible syntax combinations, or only those that do not imply a syntactical inconsistency, or the like, as considered logical practice may recommend. We proceed on the assumption that the conditions needed to avoid logical inconsistency from paradoxical inferences involving $\lambda$-abstraction are satisfied in the relevant applications. For convenience, we think of $\lambda$-abstraction equivalence naïvely as above wherever it does not threaten contradiction. The first such equivalence states:

$$
F a \leftrightarrow \lambda x[F x] a
$$

Here there occurs a qualitative expression on both sides of the biconditional, signifying that this use of $\lambda$-abstraction is not yet categorically logically reductive, let alone the expression of or justification for a corresponding ontic reduction.

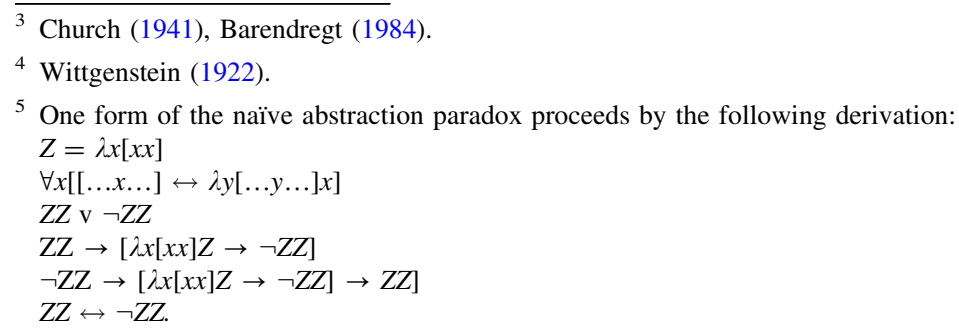


We nevertheless progress by stages from this unassuming starting place eventually to reach an explicitly relational logically equivalent formula. We note that the qualitative predication is logically equivalent to an existential predication involving a truth functional and identity relation:

$$
F a \leftrightarrow \exists x[F x \wedge x=a]
$$

Conjunction as a truth-functional relation, $\wedge$, and the identity relation, $=$, at this early stage of effecting a reductive transformation of a qualitative to a relational expression, are already incorporated in the formalization. To make the identity relation and relational expressions more explicit, we rewrite the open sentence with free variable $x$ in $x=a$ as $I x a$ or $=x a,=(x, a)$, etc. Less conventionally, we can do the same for conjunction, $\wedge$, as a truth-functional relation. ${ }^{6}$

(PUTATIVE) ONTIC 'REDUCTION' OF QUALITIES TO RELATIONS

VIA LOGICAL EQUIVALENCE

$F a \leftrightarrow \exists x[\lambda y \lambda z[F y \wedge y=z] x a]$

$\forall x[F x \leftrightarrow \exists y[\lambda z \lambda w[F z \wedge z=w] x, y]]$

Applying $\lambda$-abstraction eqivalence produces a wff explicitly containing a more generalizable two-place relational predication. From this, in turn, by a complementary abstraction, we immediately obtain a more-than-one-argument-place relational logical equivalence to the original one-argument-place qualitative predication, $\mathrm{Fa}$.

We read the order of terms in the case of $R a b$ from left to right, meaning that object $a$ has relation $R$ to $b$, rather than that $b$ has relation $R$ to $a$. By naïve $\lambda$-abstraction, the existentially bound variable ' $x$ ' takes $y$ 's place within the $\lambda$-abstraction, and constant ' $a$ ' takes $z$ 's place. The result is to derive the logically equivalent, manifestly internally relational expression, $\exists x[F x \wedge x=a]$, from which the logically equivalent two-argument-place externally relational expression is abstracted. Similarly, we interpret $\lambda$-abstracted relation $\lambda x \lambda y[R y x] b a$ as logically equivalent to $R b a$ rather than $R a b$, because of the left-to-right order in which argument places are allocated within the $\lambda$-abstraction relation term, and matching the left-to-right order of object terms satisfying the abstracted relational predicate. To say that $R a b$ under the same convention, we would need to formalize the relation by the left-to-right convention, either as $\lambda x \lambda y[R y x] a b$ or $\lambda x \lambda y[R x y] a b$.

We have seen that we can reduce qualities to relations in the sense of providing logical equivalences of qualitative to relational predications. Now we pursue the $\lambda$-abstraction reduction of qualities and relations in the opposite direction, this time

\footnotetext{
6 The reduction $F a \leftrightarrow \exists x[F x \wedge x=a]$ does not hold in free logic, where we would need to supplement the equivalence with the proposition that the name- or constant-designated object exists, $[F a \wedge E ! a] \leftrightarrow \exists x[F x \wedge x=a]$. The argument has been that even in classical logic we can syntactically reduce qualitative or one-argument-place predications to expanded relational or more-than-one-argumentplace predications, which holds true also in the free logic variant, where both $F a$ and $E ! a$ are qualitative predications, and $\exists x[F x \wedge x=a]$ in its second conjunct is relational.
} 
from relations to qualities, taking as our example a simplest standard relational predication of the form $R a b$. An adequate semantics must allow for whatever truthconditions are needed for $R a b$. The question is whether the truth-conditions for explicitly relational predications must assume the existence of relations like $R$. Are relational predications capable of being paraphrased away instead, and in that sense reduced to qualitative predications? Can we say logically exactly what we want to say when we assert that Rab, without supposing that there exists a relation $R$ ? We can do so, according to the interpretation we have been criticizing, if and only if there is a logical equivalence between $R a b$ and another one-argument-place predication. Intuitively, the equivalence is affirmed in saying that $a$ is $R$-related to $b$ if and only if $b$ has the quality of being $R$-related-to- $a$. We then have at most a relational quality, but not a quality-irreducible relation as a category unto itself. We avail ourselves of $\lambda$-abstraction devices once again in order to reduce an ostensibly relational predication to a logically equivalent syntactically qualitative predication. What are the possibilities of expressing relations as qualities in a sufficiently enriched logical notation supplemented by abstraction devices?

The difference between the relevant qualitative and relational predications is syntactically whether the predicate in question is satisfied by one or more than one argument, completed by one or more than one constant or quantifier-bound variable. We introduce the following logical equivalence, whereby the difference between qualities and relations appears to amount to nothing more or less than a stylistic preference for one mode of expression rather than another. The equivalence states:

(PUTATIVE) ONTIC 'REDUCTION' OF RELATIONS TO QUALITIES

VIA LOGICAL EQUIVALENCE

$R a b \leftrightarrow \lambda x[R a x] b$

$\forall x_{1} \ldots x_{n}\left[R x_{1} \ldots x_{n-1} x_{n} \leftrightarrow \lambda y_{1} \ldots y_{n-1}\left[R y_{1} \ldots y_{n-1}\right] x_{n}\right]$

We merely rename the abstracted property $\lambda x[R a x]=F$ (the relational quality of being $R$-related-to- $a$ ), in order subsequently to derive:

$$
R a b \leftrightarrow F b
$$

where ontic reductions of qualities to and from relations are considered, there is not merely a conditional relation, but a logical equivalence, between qualitative and relational predications. Invoking the previous definition of syntactical to ontic reduction, it follows logically both that:

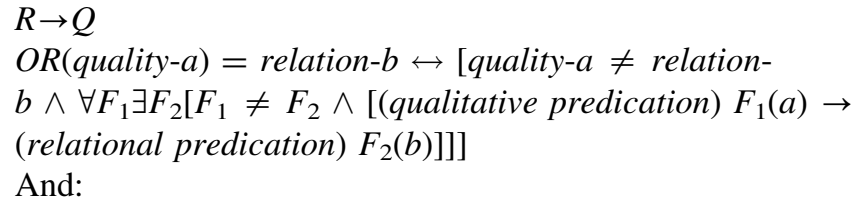




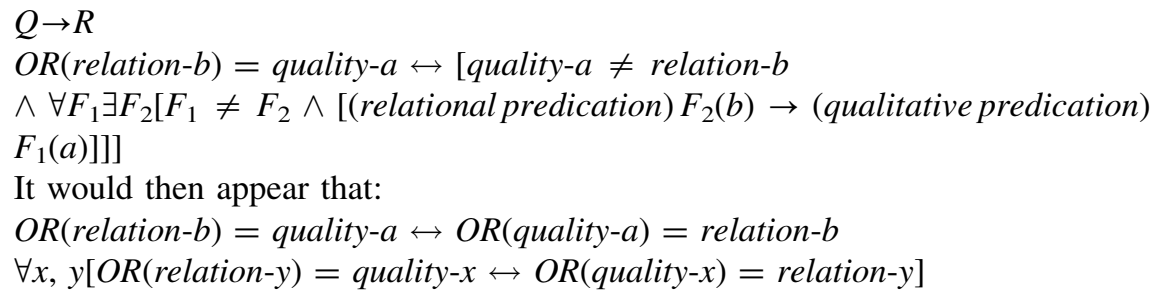

Finally, returning to the inference of the central argument, representing in functional notation the logical equivalence of qualitative and relational predications:

$\forall x \exists y[$ OR $($ relation $-x)=$ quality $-y] \wedge \forall x \exists y[$ OR $($ quality $-x)=$ relation $-y]$

As proved in the case of proposition $p=$ All triangles have 4 sides, and $q=A l l$ squares have 5 sides, we know in general that: $\neg \forall x, y[[x \leftrightarrow y] \rightarrow x=y]$. What, then, of the specific case in which $p=F a$, and $q=\exists x[\lambda y \lambda z[F y \wedge y=z] x a]$, or where $p=R a b$, and $q=\lambda x[R a x] b$ ? Are these reductive instances, where ontically $p=q$, simply because logically $p \leftrightarrow q$ ? Or are they like the above counterexample, in which truth functional equivalence is not sufficient for propositional identity, syntactically or semantically? If qualitative and relational predications are identical by virtue of being logically equivalent, then we may merely have two different ways, qualitative and relational, of predicating a property to an object or objects. Ontically, we would have only the one existent property rather than both a quality and a relation in the metaphysics' minimal ontology and logic's minimal semantic domain. If the propositions are the same, then there would seem to be good grounds for concluding that the corresponding qualities and relations are also ontically identical, although capable of syntactically distinct logically equivalent predication forms.

\section{Ontic Implications of Quality-Relation Logical Equivalences}

The truth of a logical equivalence between qualitative and relational predications is best explained as entailing what may at first seem to be two colliding, but on reflection perfectly compossible, ontic and logical or semantic propositions. As a contribution to the logic and metaphysics of properties, we accordingly propose:

(a) An ontology of properties, insofar as we recognize a distinction between one-argument-place and more-than-one-argument-place predications, logically must contain both qualities and relations. Wherever we try to speak of only qualities or only relations, we are trumped in our efforts at ontic parsimony by the logical equivalence of any qualitative predication with some relational predication, and conversely.

Logical equivalence remains in force regardless of the extra-logical considerations that might incline theory toward qualities as more fundamental than relations, or the reverse. In the present application, the subordination of the truths of metaphysics to the truths of logic implies that, wherever a quality is spoken of in formulating ontological principles, including the principle that there exist only 
qualities, there a logically equivalent relation is also expressed. The equivalence holds as a transcendent truth of logic, with metaphysics like everything else fully in its jurisdiction, even if the resources for such explicit discourse have been excluded from a designated logic or language expressing the metaphysics of properties, and in spite of the agreed upon fact that ontic commitments are always made by the acceptance of certain propositions. The same applies for the contrary proposal that there exist only relations, any mention of which is logically equivalent to a corresponding qualitative predication.

The logical equivalence of qualitative and relational predications can then alternatively be interpreted as logically implying that there are qualities wherever there are relations, and vice versa. It follows that neither category of qualities nor relations can rightly be considered as ontically more primitive or foundational than the other, and neither qualities nor relations can be made the one and only ontically reductive property category. The preferred interpretation rejects both collapsing the set of all properties into the set of all qualities minus all relations, or into the set of relations with no qualities. The qualities, logically speaking, to which ontology refers, at a higher logical court of appeal than metaphysics, are already relations, no matter how we may have limited our terminology, and the relations are already qualities. As a matter of logical equivalence, of the logical necessity expressed and logically implied by a tautology, we must always have both categories of qualities and relations in the ontology of properties wherever we have either one. This conclusion also applies to 'reductive' systematizations of property ontologies that try to make do, on the basis of the logical equivalence of qualitative and relational predications, by referring only to qualities to the exclusion of relations, or to relations excluding all qualities, contrary even to the powerful principle of logically equivalent syntactical-to-ontic reduction under criticism.

Despite (a), however, the preferred interpretation of the logical equivalence of qualitative and relational predications also logically implies:

(b) Whenever we need to speak of either qualities or relations, we can choose for an unpredetermined range of extra-logical reasons to speak exclusively of qualities and never of relations, or of relations and never of qualities.

Remarkably, as with (a) above, this includes 'reductive' systematizations of property ontologies that, in the spirit of ontic reductionism, refer only to qualities to the exclusion of relations, or to relations excluding qualities, and where a reductive theory of properties mentions only qualities or only relations. The point of (a) and (b) implied by the proposed reinterpretation, as (b) advises, is that we can choose to ignore relations in favor of qualities, or qualities in favor of relations. In articulating an ontology of properties, if it suits our purposes, we can codify metaphysical principles in terms of one category of properties rather than another, by making reference exclusively to qualities rather than relations, or to relations in preference to qualities. We should be cautioned in so doing, that, as (a) asserts, we do not thereby achieve an ontic reduction of relations to qualities or qualities to relations. We cannot determine what exists or does not exist merely by electing to use a purely qualitative versus relational or relational versus qualitative predicational vocabulary. Instead, both qualitative and relational predications are logically guaranteed 
to be intersubstitutable salva veritate in any extensional context in which either choice of terminology occurs.

This is also how we might best interpret the reductive syntactical choices available as a consequence of the propositional tautology previously mentioned, $[p \rightarrow q] \leftrightarrow[\neg p \vee q]$. Here too we may choose to eliminate $\rightarrow$ in favor of $\vee$ and $\neg$ (or the reverse) in a comparatively more economical logical syntax, while recognizing on the basis of the logical equivalence that, in a sufficiently expressively rich language, whenever we have the material conditional, for example, so have we also disjunction and negation, and conversely. Entities belonging to one metaphysical category are ontically reduced to another if and only if a true logical equivalence justifies our decreasing the number of things or types of things to which a theory is ontically committed. To reduce qualities to relations or relations to qualities is literally to reduce the total ontology of properties from two subcategories, qualities and relations, exclusively to either qualities or relations, the one in some sense subsuming the other. If qualities are just limiting cases of relations, then we need only suppose that there are relations; whereas if relations are all equivalently expressible as qualitative predications, then we need only suppose that there are qualities.

The limits of logical expression as such offer a window on what is minimally needed in a metaphysics of truth conditions for true and false propositions. Logic presupposes propositions in which properties are truly or falsely (or otherwise) predicated of objects. These properties, we often assume, can be either qualities or relations, distinguished syntactically by virtue of possessing a logical form in which exactly one or more than one object term is included when the property is predicated of the object or objects in the language. As we have seen, there exist logical equivalences within classical logic union standard $\lambda$-abstraction theory by which any qualitative expression is logically equivalent to a corresponding relational expression, and conversely. There is, moreover, an apparent gain in ontic parsimony if we can justifiably reduce qualities to relations or relations to qualities in an interesting sense, for then properties generally are simply qualities or simply relations.

But which way should it go? We might take the biconditional's truth to represent a type of logical freedom. Perhaps, if we can find good extra-logical grounds for preferring one conditional pointing in one direction over the other within the equivalence, then the equivalence permits us to justify the choice of reducing qualities to relations or relations to qualities. We simply discount one of the component conditionals, one-half of the logical equivalence of qualitative and relational predications, on extra-logical grounds, reduce it logically to the other, and thereby lay the groundwork for a metaphysics of properties consisting only of qualities or only of relations. We do so, moreover, while acknowledging that logically things could go either way, in the sense that we are satisfied for reasons consistent with but external to logic itself to accept a metaphysics of properties in which qualities are collapsed into relations, or relations into qualities.

By formalizing a logical equivalence between qualitative and relational predications, we may think that we can either eliminate qualities in favor of relations or relations in favor of qualities. Logic appears to let us choose on 
extra-logical grounds whichever reduction we may prefer in advancing a more austere properties ontology. Why not, if reductions in both directions are logically authorized? Although ontology may in some sense be formally dependent on logic, we never suppose that logic alone entails any of the juicy content of ontology, whether God exists or universals, or the like. We imagine that something more substantive than logic, the stuff of metaphysics with definite commitments as to the nature of existence, must be added to logic in order to make progress in ontology. If this is true, then perhaps we could begin to make a first stand for one logically permissible foundational metaphysical truth rather than its alternative by choosing qualities as ontically more fundamental than relations, and eliminating relations except as special cases of qualities from the ontology of properties. Or just the opposite, if we decide that relations are ontically more fundamental than qualities, eliminating qualities as merely one-argument-place limiting cases of relations. Here, in either event, we say something explicit about the world, with logic to support us insofar as there exists a logical equivalence between any qualitative predication and a corresponding relational predication.

As a final objection, consider that if we interpret the logical equivalence of qualitative and relational predications as meaning that there are both qualities and relations in even the most highly reduced logical domain of properties, then we appear to be saddled with double the number of truth-makers for such predications, as opposed to adopting the ontically reductive interpretation of their logical equivalence. The answer seems to be that in such situations we have only one truthmaker, but, since the truth-maker can be expressed either as the exemplification of a quality or relation by an object or objects, it follows that our theory, analysis, or metaphysical understanding of the relevant truth-makers, where ontic commitments are determined, logically implies the existence of both qualities and relations as property subcategories.

\section{Qualities as Relations or Relations as Qualities}

We now consider a selection of the extra-logical arguments that might be given for reducing relations to qualities, rather than qualities to relations, and the reverse. It is tempting to regard qualities as logically and ontically simpler or formally or conceptually more primitive than relations, thereby favoring the reduction of relations to qualities, including relational qualities, for at least two reasons:

(1) Qualities are minimally expressible only as one-argument-place predicates, and relations are generally expressible as more-than-one-argument-place predicates. Relations so explained seem like complicated qualities, or as compressed ways of expressing ideas about the property of an object as it stands in relation to itself or more typically another object. As such, relations are ontically speaking a special subcategory of qualities.

(2) Reducing relations to qualities is syntactically more elegant than reducing qualities to relations, because it involves only one step of $\lambda$-abstraction, whereas the reduction of qualitative to relational predications appears more 
conspicuously a matter of vacuous syntax tricks, with two overlapping $\lambda$-abstractions needed to formalize an object $a$ having a certain quality $F$ as an object related to an object $a$ explicitly said to be identical or identity-related to $a$. Why would anyone bother, except for the most abstract theoretical reasons in advancing a minimal property ontology?

What weight should be given such extra-logical quasi-aesthetic considerations in preferring the reduction of relations to qualities rather than the other way around. Do we simply adopt the prettier, subjectively more elegant, or 'intuitively appealing', of the available reductions?

Where logic seems to offer a choice of alternative reductions, provided that we can find a good reason outside of logic for preferring one reduction over another, we might find it preferable also metaphysically to consider that qualities are more basic than relations, and that relational predications are just expanded more-than-oneargument place formulations of one-argument-place qualitative predications. Why not start with qualities as the simplest cases of predication, and then build up to logically more complex relations? That of itself would be one thing, but the ontic reduction proposes not merely that in our metaphysics of properties we transition logically from qualities to relations (and relations to qualities), via the intertransformability of qualitative and relational predications, but that in the process qualities (relations) ontically swallow up relations (qualities) as special cases of qualities (relations), so that in reality there are only qualities (relations).

Giving logical and ontic preference to qualities over relations, or the opposite, despite the force of (1) and (2), can be brought to doubt. For this, we need only be troubled (3) by the implication that in either case we have thereby actually reduced the logical structure of a more-than-one-argument-place predication to a condensed, precisely one-argument-place predication, when the internal logical structure of the facilitating $\lambda$-abstract essentially contains the original more-than-one-argumentplace predicate from which it is constructed. Can we so easily change what exists or does not exist merely by playing with syntax? Have we truly eliminated what is essentially relational from relational predications, and thereby purged relations generally from ontology, when the beginning more-than-one-argument-place relational content of such a predication continues to appear explicitly in the corresponding logically equivalent $\lambda$-abstracted one-argument-place quality predication? If we say that object $b$ has the quality of being $R$-related-to- $a$, then we are referring to an explicitly relational quality, and we have not totally eliminated either relations or the concept of a relation from ontology, thought and discourse, or from logic and language in the abstract.

Why, otherwise, would anyone want to formalize $F a$ as $\exists x[\lambda y \lambda z[F y \wedge y=z] x a]$, or $R a b$ as $\lambda x[R a x] b$ ? We might choose to do so in the first instance for philosophical purposes in order to reflect the thought that object $a$ exists, where the existential quantifier is extensionally interpreted as having ontic import, ranging over a domain of existing objects only, and capable in each instance of being named as individuals by means of object constants. This is not a trivial commitment, even if widely accepted by contemporary logicians, and one well worth articulating and critically evaluating in detail, because, where the same principles as above apply, we shall not 
expect the same logical equivalence to hold when $F=$ is a detective and $a=$ Sherlock Holmes (or $a=$ Zeus and $F=$ is a great meteorological god; etc.). If we want those kinds of predications to come out true, then we must modify standard issue extensional semantics for symbolic logic to permit the naming, quantification over, and true predication of properties, to nonexistent objects like Zeus and Sherlock Holmes, and Meinong's (actually, Berkeley's and Hume's) golden mountain and even more notorious round square. ${ }^{7}$

We learn from the logical equivalences of qualitative and relational predications that there may be no answer within logic to the question whether qualities and the exemplification of qualities are metaphysically more fundamental than relations and the exemplification of relations. Shall we say that relations ontically build on and are defined in terms of qualities, or the reverse? Do $A$ and $B$ exemplify the relation of being similarly red-colored because both exemplify the quality of being red, or are both $A$ and $B$ red because they bear to one another the relation of being similarly red-colored? What is the right thing to say? In asking these questions, we begin to uncover some of the deepest roots of the metaphysical realism versus nominalism controversy. Logic, by itself, unfortunately, does not answer any of these questions, but permits us at least to formulate them univocally and with unusual clarity, and to present us with a single powerful logical equivalence that, together with the assumed ontic reduction principle, simultaneously 'reduces' both qualities to relations and relations to qualities.

Taking relations as more fundamental than qualities, on the other hand, and incorporating qualities as limiting cases of relations, in some ways more obviously and immediately handles all the limitless numbers of relations, and may fit more comfortably with a relativistic standpoint in philosophy and physics. It is nevertheless hard to overlook the fact or discount the impression that there is something highly artificial about the inclusion of qualities as limiting cases of relations. To accomplish such an expansive 'reduction', we must add vacuously relational clauses to the original expression of the exemplification of a quality in order to make it formally relational, which seems suspiciously ham-fisted. The above line of argument represents a serious challenge to the proposition that manyargument-place relations are more basic because more universal than one-argumentplace qualities. What the existence then exemplifies is the possession of relations among objects, in the limiting case of an object being related to itself. Thus, we might propose:

$$
\forall x[F x \leftrightarrow \forall y[R y y \leftrightarrow F y]]
$$

The formula appears to represent an ontic reduction of quality-hood more generally to an equivalent relational form, by interposing additional informationally redundant relational argument places. If we say $F a$, that object $a$ has quality $F$, that Allen is friendly, for example, or has the quality of being friendly, then, according to the proposed equivalence, we are saying nothing other than if we were to say that Allen has the relation $R$ to Allen of Allen's being friendly, Raa.

\footnotetext{
7 Berkeley (1949-1958, Vol. II, Second Dialogue, p. 224), Hume (1975, Section II, 'Origin of Ideas', $\S 13$, p. 19). For additional references to philosophical literature about the golden mountain, see Jacquette (2009, pp. 169-203).
} 
Philosophically, we must wonder whether cooking up such 'relations' is worthwhile, in the sense of really showing that qualities can be ontically reduced to relations by the logical equivalence of certain of their predications. Can either of these reductions be justified as preferred, if, as in the semantic instability of the liar paradox, taking the liar sentence by turns as false if true, and true if false, we can always come back logically with a reduction of qualities to relations, and relations to qualities, and logically equivalent relations again, indefinitely? It seems contrived to reduce many-argument-place relational predications to one-argument-place qualitative predications, or to expand one-argument-place qualitative predications into many-argument-place relational predications, by means of $\lambda$-abstraction, as though such a reduction were logically stable, purely for the sake of gaining points for a property ontology in which there are only qualities, or the opposite, in which there are only relations.

Nor should we expect philosophical insight from an anthropological and cognitive developmental standpoint, if it turns out that thinking subjects grasp the concept of a quality before they grasp that of a relation. The stance might be further supported externally even by the argument that individual objects are first identified by a conceptualization, so that interrelationships among objects presuppose an individual object's prior possession of qualities. First, objects must have the qualities, many a logician and metaphysician might insist, by which they are identified and individuated one from another as objects. Only then, as particularized objects, can they stand in relations to other particularized objects, each possessing a distinguishing logically consistent assemblage of qualities. Such a conceptual orientation might indeed be widely or even universally shared by all human thinkers. By itself, however, empirical facts about human cognition do not settle ontic questions, unless there is a plausible reason to suppose that other nonhuman kinds of thinkers, if any should exist, could not manage their cognitive economy by giving ontic precedence to relations over qualities, regarding them both as equifoundational, or simply not admitting the distinction.

\section{Logic and Ontology of Qualities and Relations}

The proposed philosophical recommendation is to reject interpretation (I:1) of logical equivalence between qualitative and relational predications as offering us a disjunctive choice of ontically reducing qualities to relations or relations to qualities, and to adopt instead anti-reductionistic interpretation (I:2). The reasons to be offered for preferring qualities over relations in an ontology of properties are not very persuasive in the first place, and can predictably be met by countermanding justifications for preferring relations over qualities, just as when instead we happen to favor relations over qualities.

The world, on such a conception, reality as we find it and as we try to conceptualize its most basic features, might be described as glued together by the exemplification of properties, equivalently expressible as qualities or relations. What is fundamental to metaphysics in either case is an ontology of properties exemplified by objects for a domain of properties that logically includes both 
qualities and relations, alternatively and logically equivalently expressible by both one- and more-than-one-argument place predicates. Objects have qualities and stand in relations to themselves and other objects. Propertyhood, in the most general sense, an object's possession of qualities or relations, is metaphysically fundamental, even though we may have culturally evolved a special nomenclature and a corresponding grammar and logical notation for one- and more-than-one-argumentplace property predications.

To investigate the fallacy by which the logical equivalence of quality and relational predications implies that we can get along in the ontology of properties exclusively with either qualities or relations as more fundamental than the other, consider by analogy a logical formalism that asks us to accept the syntactical convention whereby a predicate term without an object term attached is a limiting case of zero-argument-place predication that can accordingly serve as a propositional symbol. This reduction accomplishes a neat syntactical economy. But does it persuade us that predicates are reducible to propositions? If by full logical equivalence we expect that a propositional symbol can be produced from a predicate merely by attaching an object term, constant or quantifier-bound variable, then we might think that this shows propositions to be logically or ontically more basic than predicates or than the properties the predicates represent. The suggestion should nevertheless be rejected, because propositions are essentially predications that attach quality or relation predicates to object terms, whether constants or quantifierbound variables, whereas only special predicates contain propositions, which must themselves in turn contain compositionally even more basic predicates.

If we try to sustain the distinction, then we can make as good a logical case, which is to say no logically discriminating case at all, for reducing qualities to relations or relations to qualities, as we could for saying that predicates are reducible to propositions in a logic that defines propositions as zero-limit argument place instances of predicates. Propositions are rightly so-called when they propose that a certain state of affairs exists, that an object has a certain quality or stands in a certain relation to itself or other objects. Propositions, therefore, and the sentences that serve as truth-vehicles to express their meanings, are in every case nothing more or less than the attribution and expression of the attribution of a property to an object or objects. As such, propositions actually presuppose predicates representing qualities or relations, without which there is nothing for thought to propose and nothing to predicate of any object in any language or symbolic logic.

\section{Metaphysically Foundational Property Exemplification}

The moral of the story is that in logic and metaphysics we should not infer that something is more basic than something else just because the relevant terms are found in logically equivalent predications. That there exists a rule-governed syntactical transformation by which definitional or truth functional logical equivalences among categories of propositional syntax enable us to derive expressions of one logical form to and from those of another, by itself does not imply that we are justified as a result in 'reducing' relations to and from qualities, as of qualities to and from relations. 
Logic does not present us with an extra-logical choice for metaphysics and the ontology of properties in which we are free to eliminate qualities in favor of relations or relations in favor of qualities, as (I:1) prescribes. When we try to interpret the logical situation in this way, we find as above that we cannot arrive at good reasons in any case for preferring to make qualities more fundamental metaphysical building blocks than relations, or to subsume qualities as limiting cases of ontically more fundamental relations. There are incommensurable pros and cons on both sides of the dispute. The idea of throwing in our lot exclusively with qualities over relations or relations over qualities in any event is doomed to failure. The most that we are permitted is to restrict our language about properties to formalizations involving exclusively qualitative or exclusively relational predications. The logical equivalences of qualitative and relational predications metalogically transcending any constrained syntax rules imply that whenever a qualitative predication is advanced, it can be replaced in any extensional context by a logically equivalent relational predication, and vice versa. It is not that qualities themselves are dispensable in favor of relations or relations in deference to qualities, but that for whatever one-argument-place quality predication we propose, there will always be a logically equivalent more-than-one-argument-place relational predication, and conversely. Logic teaches us, not that we have the choice of going in either direction, ontically reducing qualities to relations or relations to qualities, but rather that whenever we may think we have hold of a quality in metaphysics, we might just as well say that it is a relation, and that whenever we may think we have hold of a relation, we might just as well say that it is quality.

Logical equivalences of qualitative and relational predications do not go away just because we decide metaphysically on extra-logical grounds to promote qualities over relations or relations over qualities. We are logically stuck in our ontology of properties with both qualities and relations, at least insofar as we continue to distinguish qualities from relations entirely on the number of a corresponding predicate's argument places. To logically reduce our talk in one way or another on the strength of a logical equivalence is not ontically to reduce the domain of objects about which we talk, but logically to justify an equal place for all entities under any logically equivalent designation. The logical equivalence of qualitative and relational predications, without further restriction, implies that to speak of qualities is also to speak logically equivalently of relations, and that to speak of relations is also to speak equivalently of qualities. The situation in which we recognize qualitative and relational predication forms as logically equivalent and interdefinable is analogous to the choice in a Euclidean geometry of defining lines as the shortest connection of two distinct points versus defining points as the intersection of two distinct lines. We would not propose in geometry that if we define lines in terms of points that therefore points are more fundamental than lines, since we can always turn the argument around by observing that the equivalence permits us also and with equal justice to define points in terms of lines. We are equally freighted in Euclidean geometry with both points and lines as equi-fundamental, just as we are in metaphysics with both qualities and relations in the ontology of properties. And for precisely the same reason: because, respectively, the two sets of concepts are logically interdefinable. 
We can attempt to reduce our talk about properties exclusively to qualities without relations, or to relations excluding qualities. The logical equivalence of qualitative and relational expressions is nevertheless not nullified by our extralogical motivations, but, energetic reductionist rhetoric notwithstanding, implies instead that ontically we always have qualities wherever we have relations, and conversely. There are no extra-logical reasons for preferring or rejecting one-half of a logical equivalence that ontically overpowers the logical force of a logical equivalence itself. To suppose otherwise is the wrong way to think about the existence of a logical equivalence between qualitative and relational predications, and the relation of logic to ontology. The equivalence does not license us to pick and choose on extra-logical grounds one direction of the equivalence over the other, as some logically oriented ontologists seem to believe. Instead, the equivalence prevails in either case, meaning that we cannot do away with either of the equivalents. It is a theorem of predicate-quantificational logic with $\lambda$-abstraction, a tautology of that logical system, that qualitative and relational predications are logically mutually inter-derivable, and hence that logically qualities and relations are always co-present.

We thus encounter a basic form of the positive correspondence model of truth, of the truth of a proposition describing the exemplification of a quality by an object with the actual state of affairs in which the object actually exemplifies the quality. We expect a positive correspondence between the truth-condition requirements for a correct ontology and the existence conditions of the actual or any logically possible world. Actuality is actuality, and we would not have it any other way. However, we can say something more substantive when we assert that it is the actual exemplification of properties by existent objects that constitutes the actual world. In grasping the most elementary truth conditions of true and false propositions, we touch on something deeply metaphysical, an object exemplifying properties as the most basic constituent of real existence. It is the state of affairs of an object exemplifying, possessing, or its being true of the object that the object, has a particular property, quality or relation, that is the ultimate cornerstone of existence, and hence of any adequate theoretical ontology, in a complete and correct metaphysics. We can go no further when we have reached this point, the possession by an object of a property, to paraphrase the later Wittgenstein, but our spade is turned against metaphysical bedrock. ${ }^{8}$

\section{References}

Barendregt HP (1984) The lambda calculus: its syntax and semantics. North-Holland Press, Amsterdam Berkeley G (1949-1958) In: Luce AA, Jessup TE (eds) Three dialogues between Hylas and Philonous, the works of George Berkeley Bishop of Cloyne, 9 vols., vol II. Thomas Nelson \& Sons, London

\footnotetext{
${ }^{8}$ Wittgenstein (1958), §217: 'If I have exhausted the justifications [in explaining how I am able to obey a rule] I have reached bedrock, and my spade is turned. Then I am inclined to say: "This is simply what I do."/(Remember that we sometimes demand definitions for the sake not of their content, but of their form. Our requirement is an architectonic one; the definition a kind of ornamental coping that supports nothing.)'.
} 
Church A (1941) The calculi of lambda conversion. Princeton University Press, Princeton

Hume D (1975) An enquiry concerning human understanding. In: Enquiries concerning human understanding and concerning the principles of morals, Reprinted from the 1777 Edition with Introduction and Analytical Index by L.A. Selby-Bigge, third edition, with text revised and notes by P.H. Nidditch. The Clarendon Press, Oxford

Jacquette D (2009) Meditations on Meinong's golden mountain. In: Griffin N, Jacquette D (eds) Russell versus Meinong: the legacy of 'On Denoting'. Routledge, New York

Lambert K (1987) Predication and ontology. Can J Philos 17:603-614

Quine WVO (1960) Word and object. The MIT Press, Cambridge

Quine WVO (1964) Ontological reduction and the world of numbers. J Philos 61:209-216

Wittgenstein L (1922) In: Ogden CK (ed) Tractatus Logico-Philosophicus. Routledge \& Kegan Paul, London

Wittgenstein L (1958) Philosophical investigations (trans: GEM Anscombe), 3rd edn. Macmillan Publishing Co., Inc., New York 